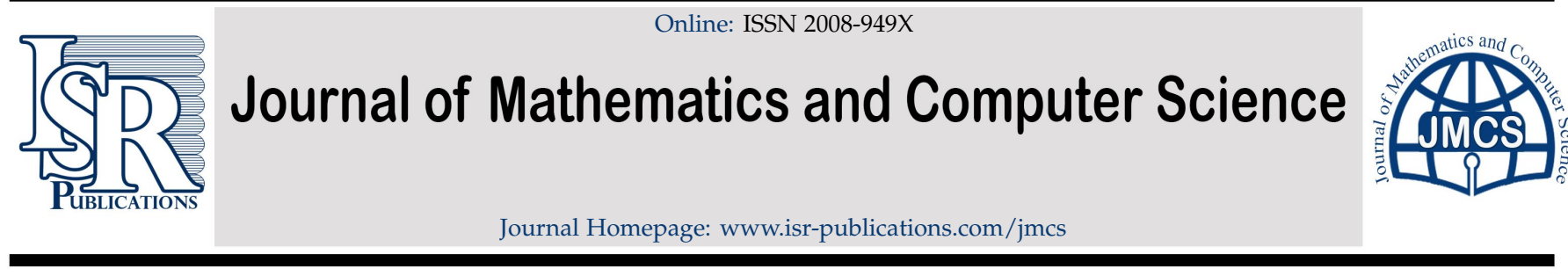

\title{
Characterization of orthogonality preserving mappings in indefinite inner product spaces
}

\author{
Sisilia Sylviani*, Hanni Garminia, Pudji Astuti
}

Algebra Research Group, Faculty of Mathematics and Natural Sciences, Institut Teknologi Bandung, Bandung, Indonesia.

\begin{abstract}
In this article we study the class of mappings in indefinite inner product spaces which preserve orthogonality. We derive characterization of orthogonality preserving mappings in indefinite inner product spaces which is a generalization of characterization of orthogonality preserving mappings in inner product spaces proposed by Chmieliński [J. Chmieliński, J. Math. Anal. Appl., 304 (2005), 158-169].
\end{abstract}

Keywords: Orthogonality preserving mappings, indefinite inner products spaces, Krein spaces.

2020 MSC: 15A86, 47B49, 47B50.

(C)2022 All rights reserved.

\section{Introduction}

This article investigates necessary and sufficient conditions for orthogonality preserving mappings in indefinite inner product spaces. Some necessary and sufficient conditions for orthogonality preserving mappings in inner product spaces were obtained by Chmieliński [2]. These results have also been successfully generalized to a certain class of orthogonality preserving mappings in sesquilinear spaces and in semi-inner product spaces [6]. In this article we extend Chmielinski's results to another direction, that is to the class of orthogonality preserving mappings in indefinite inner product spaces. A study concerning orthogonality preserving mappings in Krein spaces recently was carried out by Saraei and Amyari [4]. Some properties concerning orthogonality preserving mappings in Krein spaces were obtained, particularly relationship among several notions of orthogonality preserving. Some necessary and sufficient conditions for orthogonality preserving mappings satisfied certain conditions in Krein spaces were obtained.

Through out this article, vector spaces are always over the complex number field $\mathbb{C}$. Let $X$ be a vector space over the field $\mathbb{C}$. An indefinite inner product on $X$ is a complex value function defined on $X \times X$ that is linear with respect to the first variable, antisymmetry and nondegenerate (see e.g. [1,3]). An indefinite inner product space is a vector space over the field $\mathbb{C}$ equipped with an indefinite inner product on it. For well known terms in indefinite inner product spaces, we refer to [1] and [3]. The indefinite inner product

\footnotetext{
*Corresponding author

Email address: sisilia.sylviani@s.itb.ac.id (Sisilia Sylviani)

doi: $10.22436 /$ jmcs.026.01.02
}

Received: 2021-07-06 Revised: 2021-07-27 Accepted: 2021-08-10 
which equips an indefinite inner product space $X$ will be denoted by $[-,-]_{X}$ or in short, when it does not cause confusion, by $[-,-]$.

Let $X, Y$ be two indefinite inner product spaces. A mapping $T: X \longrightarrow Y$ is called orthogonality preserving if the following condition holds:

$$
\forall u, v \in X, u \perp v \Rightarrow \mathrm{T}(\mathrm{u}) \perp \mathrm{T}(v) .
$$

The mapping $\mathrm{T}$ is called strongly orthogonality preserving if the following condition holds:

$$
\forall \mathrm{u}, v \in \mathrm{X}, \mathrm{u} \perp v \Leftrightarrow \mathrm{T}(\mathrm{u}) \perp \mathrm{T}(v) .
$$

This article proves the following theorem.

Theorem 1.1. Let $X$ and $Y$ be two indefinite inner product spaces over $\mathbb{C}$. For a mapping $T: X \longrightarrow Y$, the following statements are equivalent.

(i) $\mathrm{T}$ is linear and there exists a nonzero real number $\gamma$ such that for all $\mathrm{u} \in \mathrm{X}$ we have $[\mathrm{T}(\mathrm{u}), \mathrm{T}(\mathrm{u})]_{Y}=\gamma[\mathrm{u}, \mathrm{u}]_{\mathrm{X}}$.

(ii) There exists a nonzero real number $\gamma$ such that for all $u, v \in X$ we have $[\mathrm{T}(\mathrm{u}), \mathrm{T}(v)]_{Y}=\gamma[\mathrm{u}, v]_{\mathrm{X}}$.

(iii) $\mathrm{T}$ is linear and strongly orthogonality preserving.

(iv) $T$ is linear, orthogonality preserving, and there exists $x \in X$ such that $[\mathrm{T}(\mathrm{x}), \mathrm{T}(\mathrm{x})]_{\mathrm{Y}} \neq 0$.

Consider that if $\mathrm{X}$ and $\mathrm{Y}$ in Theorem 1.1 above are both inner product spaces, then we obtain the results [2, Theorem 1], and also [6, Theorem 1.2]. However, we need to note that generalizing [2, Theorem $1]$ to the class of indefinite inner product spaces requires adjustment. A nonvanishing mapping in inner product spaces is equivalent to the existence of an element with the norm of its map is positive. This equivalent property is no longer valid when we are working with indefinite inner product spaces.

Example 1.2 shows that condition T nonvanishing in [2, Theorem 1] is not enough when it is extended to indefinite inner product spaces. On the contrary, Example 1.3 shows that condition $\gamma$ positive in [2, Theorem 1] needs to be weakened when it is generalized to indefinite inner product spaces.

Example 1.2. Consider $\mathbb{C}^{2}$ as an indefinite inner product space with indefinite inner product on $\mathbb{C}^{2}$ defined as $[u, v]=u_{1} v_{1}-u_{2} v_{2}$ for all $u=\left(u_{1}, u_{2}\right)^{\mathfrak{t}}, v=\left(v_{1}, v_{2}\right)^{\mathfrak{t}} \in \mathbb{C}^{2}$. The nonvanishing linear operator on $\mathbb{C}^{2}$ defined as $T(u)=\left(u_{1}+u_{2}\right)(1,1)^{t}$ for all $u=\left(u_{1}, u_{2}\right)^{t} \in \mathbb{C}^{2}$ is orthogonality preserving. However $T$ does not satisfy Theorem 1.1.

Example 1.3. Again, consider the indefinite inner product space $\mathbb{C}^{2}$ defined in Example 1.2. The rotation operator on $\mathbb{C}^{2}$ defined as $R(u)=\left(-u_{2}, u_{1}\right)^{t}$ for all $u=\left(u_{1}, u_{2}\right)^{t} \in \mathbb{C}^{2}$ is orthogonality preserving. $R$ satisfies Theorem 1.1 with $\gamma=-1$, it is not positive.

\section{Proof of Theorem 1.1}

The proof of the implications (i) $\Rightarrow$ (ii) and (iii) $\Rightarrow$ (iv) of [2, Theorem 1] can be directly extended to obtain the same implications of Theorem 1.1. For the implication (ii) $\Rightarrow$ (iii), let (ii) holds. The strong orthogonality preserving property of $T$ and the existence of $x \in X$ such that $[T(x), T(x)]_{Y} \neq 0$ in (iii) can be obtained directly form (ii). The proof of $T$ being linear in [2, Theorem 1 (iii)] can be applied to obtain $T$ linear in Theorem 1.1 after we can show that $\operatorname{Im}(T)$ is non-degenerate. Let $T(u) \in \operatorname{Im}(T)$ for some $u \in X$ with the property $[\mathrm{T}(\mathrm{u}), \mathrm{T}(v)]_{Y}=0$ for all $\mathrm{T}(v) \in \operatorname{Im}(\mathrm{T})$. Applying (ii) we obtain $[u, v]_{X}=0$ for all $v \in X$ which, together with the non-degenerate property of $X$, implies $u=0$. Hence $T(u)=0$. $\operatorname{Thus} \operatorname{Im}(T)$ is non-degenerate and hence $T$ is linear.

Thus far, it remains to prove the implication (iv) $\Rightarrow$ (i) and it is shown in Lemma 2.4 below. Before that, we discuss some properties and results concerning Krein spaces which will be utilized. A Krein space 
$\mathrm{K}$ is an indefinite inner product space which can be decomposed as $\mathrm{K}=\mathrm{K}^{+} \oplus \mathrm{K}^{-}$for some subspaces $\mathrm{K}^{+}$and $\mathrm{K}^{-}$satisfying the following conditions: $\mathrm{K}^{+}$with respect to $[-,-]_{\mathrm{K}}$ is an Hilbert space, $\mathrm{K}^{-}$with respect to $-[-,-]_{\mathrm{K}}$ is an Hilbert space and $\mathrm{K}^{+} \perp \mathrm{K}^{-}$(see e.g. [1,3]). The explained representation of a Krein space $\mathrm{K}=\mathrm{K}^{+} \oplus \mathrm{K}^{-}$is called a canonical decomposition of $\mathrm{K}$. Any finitely dimensional indefinite inner product space is Krein space. Hence, for any finitely dimensional inner product space we have an orthonormal basis obtained from a canonical decomposition of it.

Lemma 2.1. Let $\mathrm{T}: \mathrm{X} \rightarrow \mathrm{Y}$ be an orthogonality preserving linear mapping for some $\mathrm{X}$ an inner product space and $\mathrm{Y}$ an indefinite inner product space. If there exists $\mathrm{x} \in \mathrm{X}$ such that $[\mathrm{T}(\mathrm{x}), \mathrm{T}(\mathrm{x})] \neq 0$, then $\operatorname{Im}(\mathrm{T})$ forms an inner product space either with respect to $[-,-]_{Y}$ or $-[-,-]_{Y}$.

Proof. It is enough to show the case if $[T(x), T(x)]_{Y}>0$ then $\operatorname{Im}(T)$ with respect to $[-,-]_{Y}$ forms an inner product space. So, assume there exists $x \in X$ such that $[T(x), T(x)]_{Y}>0$. Since $T$ is linear we obtain $x \neq 0$. We can conclude that $\operatorname{Im}(T)$ with respect to $[-,-]_{Y}$ forms an inner product space if we can show that for any $y \in \operatorname{Im}(T), y \neq 0,[y, y]_{Y}>0$.

Let $y \in \operatorname{Im}(T)$ with $y \neq 0$. Let $u \in X$ such that $y=T(u)$. If $u=\alpha x$ for some $\alpha \neq 0$, clearly

$$
[y, y]_{Y}=|\alpha|^{2}[T(x), T(x)]_{Y}>0 .
$$

So, suppose $u \notin \operatorname{Span}\{x\}$. We have $u=u_{1}+\alpha x$ for some $\alpha \in \mathbb{C}, \mathfrak{u}_{1} \in X, \mathfrak{u}_{1} \neq 0$ and $\mathfrak{u}_{1} \perp x$. Choose $\mathfrak{u}_{2}=\beta u_{1}$, for some $\beta \in \mathbb{C}, \beta \neq 0$ such that $\left[u_{2}, u_{2}\right]_{X}=[x, x]_{X}$. We obtain $u_{2} \perp x$. Hence $\left[T\left(u_{2}\right), T(x)\right]_{Y}=0$. Further,

$$
\left[\mathfrak{u}_{2}+x, u_{2}-x\right]_{X}=\left[u_{2}, u_{2}\right]_{X}-[x, x]_{X}=0,
$$

which implies

$$
\left[T\left(u_{2}\right)+T(x), T\left(u_{2}\right)-T(x)\right]_{Y}=0 .
$$

As a result, we obtain

$$
\left[\mathrm{T}\left(\mathrm{u}_{2}\right), \mathrm{T}\left(\mathrm{u}_{2}\right)\right]_{\mathrm{Y}}=[\mathrm{T}(\mathrm{x}), \mathrm{T}(\mathrm{x})]_{\mathrm{Y}}>0 .
$$

So,

$$
\begin{aligned}
\mathrm{Y} & =[\mathrm{T}(\mathrm{u}), \mathrm{T}(\mathrm{u})]_{\mathrm{Y}} \\
& =\left[\mathrm{T}\left(\beta^{-1} \mathrm{u}_{2}+\alpha x\right), \mathrm{T}\left(\beta^{-1} \mathfrak{u}_{2}+\alpha x\right)\right]_{Y} \\
& =\left|\beta^{-1}\right|^{2}\left[\mathrm{~T}\left(\mathrm{u}_{2}\right), \mathrm{T}\left(\mathrm{u}_{2}\right)\right]_{Y}+|\alpha|^{2}[\mathrm{~T}(x), \mathrm{T}(x)]_{Y}>0 .
\end{aligned}
$$

Lemma 2.2. Let $\mathrm{K}$ be a Krein space and $\mathrm{x} \in \mathrm{K}, \mathrm{x} \neq 0$ with $[\mathrm{x}, \mathrm{x}] \neq 0$. Then there exists a canonical decomposition $\mathrm{K}=\mathrm{K}^{+} \oplus \mathrm{K}^{-}$such that $\mathrm{x} \in \mathrm{K}^{+}$if $[\mathrm{x}, \mathrm{x}]>0$, otherwise $\mathrm{x} \in \mathrm{K}^{-}$.

Proof. It is enough to prove the lemma for the case $[x, x]>0$. So Let $[x, x]>0$. Let $K=K^{+} \oplus K^{-}$be a canonical decomposition of $K$. If $x \in K^{+}$then the proof is complete. Hence, suppose $x=x^{+}+x^{-}$for some $x^{+} \in K^{+}, x^{-} \in K^{-}$and $x^{-} \neq 0$. Since $[x, x]=\left[x^{+}, x^{+}\right]+\left[x^{-}, x^{-}\right],\left[x^{-}, x^{-}\right]<0$ and $[x, x]>0$, then $x^{+} \neq 0$ and $\left[x^{+}, x^{+}\right]>\left|\left[x^{-}, x^{-}\right]\right|>0$. Define

$$
\begin{aligned}
& \mathrm{L}^{+}=\left\{y \in \mathrm{K}^{+} \mid\left[y, x^{+}\right]=0\right\}, \\
& \mathrm{L}^{-}=\left\{y \in \mathrm{K}^{-} \mid\left[y, x^{-}\right]=0\right\} .
\end{aligned}
$$

In the framework of Hilbert spaces, we obtain that $\mathrm{L}^{+}$and $\mathrm{L}^{-}$are the orthogonal complement subspaces of respectively $\operatorname{Span}\left(\mathrm{x}^{+}\right)$in $\left(\mathrm{K}^{+},[-,-]\right)$and $\operatorname{Span}\left(\mathrm{x}^{-}\right)$in $\left(\mathrm{K}^{-},-[-,-]\right)$. Hence

$$
\mathrm{K}^{+}=\operatorname{Span}\left(\mathrm{x}^{+}\right) \oplus \mathrm{L}^{+}, \quad \text { and } \quad \mathrm{K}^{-}=\operatorname{Span}\left(\mathrm{x}^{-}\right) \oplus \mathrm{L}^{-} \text {. }
$$


Define $S=\operatorname{Span}\left(x^{+}, x^{-}\right)$. We obtain $x \in S$, and $K=\mathrm{L}^{+} \oplus \mathrm{S} \oplus \mathrm{L}^{-}$with $\mathrm{L}^{+}, \mathrm{L}^{-}, \mathrm{S}$ are pairwise orthogonal. We define $y=\alpha x^{+}+x^{-} \in S$ with $\alpha=-\frac{\left[x^{-}, x^{-}\right]}{\left[x^{+}, x^{+}\right]}$. Utilizing the fact $\left[x^{+}, x^{+}\right]>0,\left[x^{-}, x^{-}\right]<0$ and $[x, x]>0$ we obtain

$$
\begin{aligned}
{[y, x] } & =\alpha\left[x^{+}, x^{+}\right]+\left[x^{-}, x^{-}\right]=0, \\
{[y, y] } & =\left[\alpha x^{+}+x^{-}, \alpha x^{+}+x^{-}\right] \\
& =\alpha^{2}\left[x^{+}, x^{+}\right]+\left[x^{-}, x^{-}\right] \\
& =\frac{\left[x^{-}, x^{-}\right]^{2}}{\left[x^{+}, x^{+}\right]}+\left[x^{-}, x^{-}\right] \\
& =\frac{\left[x^{-}, x^{-}\right]}{\left[x^{+}, x^{+}\right]}\left(\left[x^{-}, x^{-}\right]+\left[x^{+}, x^{+}\right]\right) \\
& =\frac{\left[x^{-}, x^{-}\right]}{\left[x^{+}, x^{+}\right]}[x, x]<0 .
\end{aligned}
$$

Next, define $\mathrm{K}_{1}^{+}=\mathrm{L}^{+}+\operatorname{Span}(\mathrm{x}), \mathrm{K}_{1}^{-}=\mathrm{L}^{-}+\operatorname{Span}(\mathrm{y})$. Then, it is routine to show $\mathrm{K}=\mathrm{K}_{1}^{+} \oplus \mathrm{K}_{1}^{-}$is a canonical decomposition of $\mathrm{K}$ with $x \in \mathrm{K}_{1}^{+}$.

Lemma 2.3. Let $\mathrm{T}: \mathrm{X} \rightarrow \mathrm{Y}$ be an orthogonality preserving linear mapping where $\mathrm{X}$ is a Krein space and $\mathrm{Y}$ is an indefinite inner product space. If there exists $\mathrm{x} \in \mathrm{X}$ such that $[\mathrm{T}(\mathrm{x}), \mathrm{T}(\mathrm{x})]_{\mathrm{Y}} \neq 0$ then there exists a non-zero real number $\gamma$ such that for all $\mathrm{u} \in \mathrm{X},[\mathrm{T}(\mathrm{u}), \mathrm{T}(\mathrm{u})]_{\mathrm{Y}}=\gamma[\mathrm{u}, \mathrm{u}]_{\mathrm{X}}$.

Proof. Without lost of generality we can assume $x \in X$ in the theorem satisfies conditions $[x, x]_{X}>0$ and $[T(x), T(x)]_{Y}>0$. Based on Lemma 2.2, $X$ has a canonical decomposition $X=X^{+} \oplus X^{-}$where $x \in X^{+}$. Consider the restriction $T$ on the subspace $X^{+}$, i.e., $\left.T\right|_{X^{+}}: X^{+} \rightarrow Y$. According to Lemma 2.1, the indefinite inner product $[-,-]_{Y}$ on $T\left(X^{+}\right)$is an inner product. As a result, the restriction of $T$ on the subspace $X^{+}$ $\left.\mathrm{T}\right|_{X^{+}}: X^{+} \rightarrow \mathrm{T}\left(X^{+}\right)$is a non-vanishing orthogonality preserving linear mapping from the inner product space $X^{+}$to the inner product space $T\left(X^{+}\right)$. Referring to [2, Theorem 1] there exists $\gamma>0$ such that for all $u \in X^{+},[T(u), T(u)]_{Y}=\gamma[u, u]_{X}$.

Let $u \in X^{-}, u \neq 0$. We have $[u, u]_{X}<0$. Take a vector $v \in X^{+}$with square norm $[v, v]_{X}=-[u, u]_{X}$. Write $y=u+v \in X$. We obtain

$$
[y, y]_{X}=[u, u]_{X}+[v, v]_{X}=0 .
$$

Hence, utilizing the orthogonality preserving property of $\mathrm{T}$, we obtain $[\mathrm{T}(\mathrm{u}), \mathrm{T}(v)]_{Y}=0$ and $[\mathrm{T}(\mathrm{y}), \mathrm{T}(\mathrm{y})]_{\mathrm{Y}}$ $=0$. As a result

$$
0=[\mathrm{T}(\mathrm{y}), \mathrm{T}(\mathrm{y})]_{\mathrm{Y}}=[\mathrm{T}(\mathrm{u}), \mathrm{T}(\mathrm{u})]_{\mathrm{Y}}+[\mathrm{T}(\mathrm{v}), \mathrm{T}(\mathrm{v})]_{\mathrm{Y}},
$$

then

$$
[\mathrm{T}(\mathrm{u}), \mathrm{T}(\mathrm{u})]_{\mathrm{Y}}=-[\mathrm{T}(v), \mathrm{T}(v)]_{Y}=-\gamma[v, v]_{X}=\gamma[\mathrm{u}, \mathrm{u}]_{X} .
$$

Let $u \in X$. We have $y=u^{+}+u^{-}$for some $u^{+} \in X^{+}$and $u^{-} \in X^{-}$. Employing the properties $u^{+} \perp u^{-}$, orthogonality preserving of $\mathrm{T}$ and the above obtained facts, we obtain

$$
\begin{aligned}
{[\mathrm{T}(\mathrm{u}), \mathrm{T}(\mathrm{u})]_{\mathrm{Y}} } & =\left[\mathrm{T}\left(\mathrm{u}^{+}\right), \mathrm{T}\left(\mathrm{u}^{+}\right)\right]_{\mathrm{Y}}+\left[\mathrm{T}\left(\mathrm{u}^{-}\right), \mathrm{T}\left(\mathrm{u}^{-}\right)\right]_{\mathrm{Y}} \\
& =\gamma\left[\mathrm{u}^{+}, \mathrm{u}^{+}\right]_{\mathrm{X}}+\gamma\left[\mathrm{u}^{-}, \mathrm{u}^{-}\right]_{\mathrm{X}} \\
& =\gamma[\mathrm{u}, \mathrm{u}]_{\mathrm{X}} .
\end{aligned}
$$

Lemma 2.4. Let $\mathrm{T}: \mathrm{X} \rightarrow \mathrm{Y}$ be an orthogonality preserving linear mapping where $\mathrm{X}$ and $\mathrm{Y}$ are indefinite inner product spaces. If there exists $\mathrm{x} \in \mathrm{X}$ such that $[\mathrm{T}(\mathrm{x}), \mathrm{T}(\mathrm{x})] \neq 0$ then there exists a non-zero real number $\gamma$ such that for all $\mathrm{u} \in \mathrm{X},[\mathrm{T}(\mathrm{u}), \mathrm{T}(\mathrm{u})]_{\mathrm{Y}}=\gamma[\mathrm{u}, \mathrm{u}]_{\mathrm{X}}$. 
Proof. Let $x \in X$ satisfies $[T(x), T(x)]_{Y} \neq 0$. Since $T$ is linear and $T$ preserves orthogonality then $x \neq 0$ and $[x, x]_{X} \neq 0$. Write $\gamma=\frac{[T(x), T(x)]_{Y}}{[x, x]_{X}}$. It is trivial that $[T(x), T(x)]_{Y}=\gamma[x, x]_{X}$.

We will show that for any $u \in X$ the following equation holds:

$$
[\mathrm{T}(\mathrm{u}), \mathrm{T}(\mathrm{u})]_{\mathrm{Y}}=\gamma[\mathrm{u}, \mathrm{u}]_{X} \text {. }
$$

Let $u \in X$. We consider four cases. The first case is when $u \in \operatorname{Span}(x)$. Using the linearity of $T$ it can be shown that $u$ satisfying (2.1). The second case is when $u \notin \operatorname{Span}(x)$ but $[u, u]_{X}=0$. For this case, applying the orthogonality preserving of $T$, we have $u$ satisfying (2.1).

The third case is when $u \notin \operatorname{Span}(x),[u, u]_{x} \neq 0$ but the subspace $S=\operatorname{Span}(x, u)$ degenerate. Let $v \in S, v \neq 0$ such that $[v, w]_{X}=0$ for all $w \in S$. Since $\{x, u\}$ is a basis of $S$, let $v=\alpha x+\beta u$ for some $\alpha, \beta \in \mathbb{C}$. It is trivial that both $\alpha \neq 0$ and $\beta \neq 0$. Hence, we have $\{x, v\}$ is also a basis of $S$. Let $u=\mu x+v v$ for some $\mu, v \in \mathbb{C}$. Since $v$ is degenerate, we obtain $[u, u]_{X}=|\mu|^{2}[x, x]_{X}$. Applying the linearity and the orthogonality preserving of $\mathrm{T}$ we also obtain

$$
\begin{aligned}
Y= & |\mu|^{2}[T(x), T(x)]_{Y}+\mu \bar{v}[T(x), T(v)]_{Y}+v \bar{\mu}[T(v), T(x)]_{Y} \\
& +|v|^{2}[T(v), T(v)]_{Y} \\
= & |\mu|^{2}[T(x), T(x)]_{Y} \\
= & \gamma|\mu|^{2}[x, x]_{X} \\
= & \gamma[u, u]_{X} .
\end{aligned}
$$

Thus $u$ satisfies (2.1).

The fourth and the last case is when $u \notin \operatorname{Span}(x),[u, u]_{x} \neq 0$ and the subspace $S=\operatorname{Span}(x, u)$ nondegenerate. For this case we have $S$ is a Krein space. Consider the restriction $T$ on the subspace $S$, i.e., $\left.T\right|_{S}: S \longrightarrow Y$, with $S$ is a Krein space, and there exists $x \in S$ such that $[T(x), T(x)]_{Y} \neq 0$. According to Lemma 2.3 there exists a nonzero real number $\delta$ such that

$$
[\mathrm{T}(w), \mathrm{T}(w)]_{Y}=\delta[w, w]_{\mathrm{X}}, \quad \forall w \in \mathrm{S}
$$

Particularly, we have $\left[T(x), T(x)_{Y}=\delta[x, x]_{X}\right.$. Since $[x, x]_{X} \neq 0$ and referring to the definition of $\gamma$ above, we have $\delta=\gamma$. Thus, $u$ satisfies (2.1).

\section{Concluding remarks}

In this article we have been able to expand the results concerning necessary and sufficient conditions for orthogonality preserving mappings on inner product spaces to indefinite inner products spaces. Meanwhile, recently, Saraei and Amyari [5] have been able to expand the result concerning approximately orthogonality preserving maps on inner products spaces to Krein spaces, a certain subclass of indefinite inner product spaces. This fact raises an interesting open question whether those expansion can go further to indefinite inner product spaces.

\section{Acknowledgment}

This work was supported by Program Penelitian Pengabdian kepada Masyarakat dan Inovasi 2020 of Institut Teknologi Bandung, Indonesia. 


\section{References}

[1] J. Bognar, Indefinite Inner Product Spaces, Springer-Verlag, New York-Heidelberg, (1974). 1, 2

[2] J. Chmieliński, Linear mappings approximately preserving orthogonality, J. Math. Anal. Appl., 304 (2005), 158-169. 1, $1,2,2$

[3] I. Gohberg, P. Lancaster, L. Rodman, Indefinite Linear Algebra and Applications, Birkhäuser Verlag, Basel, (2005). 1, 2

[4] A. Saraei, M. Amyari, Orthogonality Preserving Mappings in Krein Spaces, J. Math. Anal., 10 (2019), 112-122. 1

[5] A. Saraei, M. Amyari, Approximately orthogonality preserving maps in Krein spaces, Aequationes Math., 94 (2020), 137-149. 3

[6] P. Wójcik, Operators preserving sesquilinear form, Linear Algebra Appl., 469 (2015), 531-538. 1, 1 